\title{
THE MODE OF ACTION OF CONTRACTILE EFFECTS INDUCED BY EXTERNAL CALCIUM AND ITS RELATED BIVALENT CATIONS IN THE KCl-DEPOLARIZED RAT UTERUS
}

\author{
Masayasu KIMURA, Ikuko KIMURA and Mutsuko MAEKAWA \\ Department of Chemical Pharmacology, Faculty of Pharmaceutical Sciences, \\ Toyama Medical and Pharmaceatical University, Sagitani, Toyama 930-01, Japan
}

Accepted February 28, 1978

\begin{abstract}
The cumulative log dose-response curve (DRC) isometrically produced by $\mathrm{CaCl}_{2}$ on depolarized uteri of rats in $\mathrm{Ca}^{2+}$-free $\mathrm{KCl}$ Ringer's solution ( $\mathrm{pH} 7.8$ ) showed a plateau-shaped type, when responses were plotted as a percent of the maximal tension of $50 \mu \mathrm{M}$ acetylcholine, whereas those produced by $\mathrm{BaCl}_{2}$ or $\mathrm{SrCl}_{2}$ were a simple sigmoid type with the maximal response near the height of the plateau induced by $\mathrm{Ca}^{2 *}$. The platcau phase of $\mathrm{Ca}^{2}$ was inhibited competitively by $\mathrm{Mg}^{2+}(0.5-50 \mathrm{mM})$ and non-competitively by $\mathrm{Mn}^{3+}(30 \mu \mathrm{M}-1 \mathrm{mM})$, whereas the maximal contraction of $\mathrm{Ca}^{2+}$ was not inhibited by either ion. Dantrolene $(0.1 \mathrm{mM})$ inhibited both the plateau and the maximal contraction of $\mathrm{Ca}^{2}$ and retained the plateau-shaped type. EGTA ( $2 \mathrm{mM}$ ) potentiated the plateau to the maximal response level and changed the DRC induced by $\mathrm{Ca}^{2+}$ to the simple sigmoid type. From these results, the plateau of DRC induced by $\mathrm{Ca}^{2-}$ seems to be mainly due to actions of external $\mathrm{Ca}^{2+}$ on the calcium binding of internal surface of cell membrane, suggesting that the internal surface is the site of action of the bivalent cations tested.
\end{abstract}

The contractile mechanism of smooth muscle, like that of striated muscle, is activated by calcium ions although the mechanism operating in smooth muscle is less well understood than in striated muscle. When drugs induce contraction in smooth muscle, such may be indirect interactions with calcium ions, either by activating intracellular calcium stores or by allowing for an increased influx of external calcium ions.

Depolarized smooth muscles respond by contraction to low concentrations of external calcium ions. Thus it was found by Schild (1) that concentrations of 1-2//M calcium chloride added to "calcium-free" potassium Ringer's solution induced threshold contractile responses in the depolarized rat uterus. Magnesium ion interacts with the contractile responses of calcium ion in the depolarized rat uterus. Schild (1) reported that magnesium ions had at least three actions in the depolarized rat uterus; 1) an irregular contractile effect of its own which exhibits rapid tachyphylaxis; 2) competitive antagonism of the contractile action of low and medium concentrations of calcium ions; and 3) potentiation of the contractile action of high and maximal concentrations of calcium ions, increasing up to $25 \%$ the maximal tension attainable with calcium ions only.

The mechanism of action of magnesium ion in depolarized smooth muscle has not been studied in detail and the primary aim of the present study was to determine the interaction between calcium or its related bivalent cations and magnesium ion in this preparation. During studies on the mode of action of calcium ion in the $\mathrm{KCl}$-depolarized uterus, we 
unexpectedly found that the dose-response curve of calcium ion has a plateau-shape consisting of two steps, when responses were plotted as percent for the maximal tension of 50, $/ \mathrm{M}$ acetylcholine $(\mathrm{ACh})$ on the non-depolarized uterus. An analysis of the log doseresponse curve induced by calcium ion under the influence of manganese ion, diliazem, dantrolene and EGTA is reported herein.

\section{MATERIALS AND METHODS}

The methods were similar to those reported by Schild (2). Female, virgin Wistar rats weighing 190-200 g were sacrificed at diestrus and the excised uteri were suspended in a jacketed and temperature-controlled isolated organ bath of $6 \mathrm{ml}$ volume normally kept at $30^{\circ} \mathrm{C}$. Isometric tension on the uterus was measured by Swema (SG 4/3) strain gauge transducers or by force displacement transducers SB-1T (Nihon Kohden), and recorded on an ink-writing polygraph. The transducers were joined to screw stands to which a verniertype sliding scale was also attached and such enabled length changes of the uterus to be measured to the nearest $0.1 \mathrm{~mm}$. Two horns of the same uterus were usually investigated in parallel, and an automatic assay apparatus was employed (3), early in this study in the laboratory of Schild.

The uterus was immersed in Tyrode solution and then stimulated with 1 " $\mathrm{M}$ ACh until constant responses were produced. The maximal force exerted by one horn was in the range of 2-6 g with $50 \mu \mathrm{M}$ ACh. Immersion of the whole uterus in potassium Ringer's solution produced a rapid near-maximal contraction followed at once by relaxation which levelled off exponentially to a new base-line in the course of 5-15 min. If tension was relieved at this point the muscle was seen to have shortened considerably. The following procedure was used for studying drug effects in the Tyrode solution; the amplification of the tension record was usually increased up to five-fold. The muscle was kept at a basic tension of 0.25-0.6 g (about $10 \%$ of maximal ACh tension). Tension had to be frequently readjusted as the depolarized uterus underwent slow changes in length in the course of prolonged experiments usually in the sense of shortening; it also underwent length changes after each wash. After depolarization with Ca-free KCl Ringer's solution, the muscle was stimulated intermittently by calcium, barium or strontium ions. The effects of magnesium ion, manganese ion, diltiazem and dantrolene were expressed as the measured difference of isometric tension induced by calcium, barium or strontium ions in the presence and in the absence of these agents. The effects of EGTA or EDTA were studied as follows; after pretreatment with EGTA or EDTA solution for $30 \mathrm{~min}$, the uterine segments were washed out with Cafree $\mathrm{KCl}$ Ringer's solution for $20 \mathrm{~min}$. The log dose-response curves induced by calcium, barium or strontium cations were usually measured cumulatively, but in some experiments single doses were administered at random. When using both procedures, the data were similar, therefore the average was calculated.

The depolarizing medium used was Ca-free $\mathrm{KCl}$ Ringer's solution $(\mathrm{mM}: 145 \mathrm{KCl}, 12$ $\mathrm{KHCO}_{3}$ and 6 glucose) aerated with $95 \% \mathrm{O}_{2}$ and $5 \% \mathrm{CO}_{2}$. The pH of the aerated Ringer's solution was about 7.8. As calculated from measurements of conductivity of the analytical 
reagents (BHC) used, the calcium content of $\mathrm{KCl}$ Ringer's solution was always less than $10 \mu \mathrm{M}$.

Drugs used in these studies were ACh (Daiichi), calcium chloride, barium chloride, strontium chloride, EDTA (Wako), manganese chloride, EGTA (Nakarai), diltiazem (Tanabe) and dantrolene sodium (Yamanouchi). All were dissolved in distilled water for concentrations tested. EGTA solution consisted of $\mathrm{K}_{2} \mathrm{SO}_{4} 60 \mathrm{mM}, \mathrm{MgSO}_{4} 4 \mathrm{mM}$, EGTA $2 \mathrm{mM}$, Tris $20 \mathrm{mM}$ and maleic acid $20 \mathrm{mM}\left(\mathrm{pH} 8.0\right.$ ). EDTA solution consisted of $\mathrm{K}_{2} \mathrm{SO}_{4}$ $60 \mathrm{mM}$, EDTA-2Na $3 \mathrm{mM}$, Tris $20 \mathrm{mM}$ and maleic acid (pH 8.0).

\section{RESULTS}

Log dose-response curves induced by calcium, barium and strontium cations: The rat uterus immersed in Ca-free $\mathrm{KCl}$ Ringer's solution at $30^{\circ} \mathrm{C}$ is comparatively quite sensitive to external calcium ions. The record of tension developed with $1 \mu \mathrm{M}$, as this dose is the lowest dose which elicited response with external calcium chloride, and attained to submaximal response with $1 \mathrm{mM}$ (1). At the concentration of over $1 \mathrm{mM}$ of external calcium ion, the increase of tension was observed following the plateau of maximal response. In Fig. 1, the log dose-response relations of external calcium or barium ions were plotted for the $\mathrm{KCl}$-depolarized uterus and compared with those of $\mathrm{ACh}$ or barium chloride in a normal, non-depolarized uterus in order to calculate the tension induced by $50 \mu \mathrm{M} \mathrm{ACh}$ for the maximal response.

The main findings were as follows; the contractile response induced by influx of external calcium ion in $\mathrm{Ca}$-free $\mathrm{KCl}$ depolarized medium was considerably weaker than that of intracellular calcium released by ACh depolarization. If the minimal dose $1 \mathrm{nM} A \mathrm{ACh}$ cor-

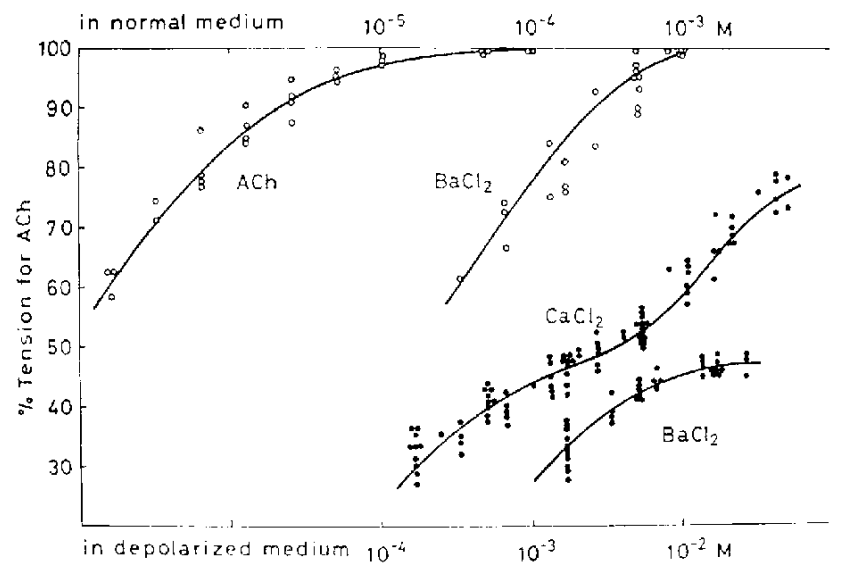

Fig. 1. Log dose-response curves induced by $\mathrm{Ca}^{2+}$ and $\mathrm{Ba}^{2+}$ in $\mathrm{Ca}$-free $\mathrm{KCl}$-depolarized medium (O), as compared with those of acetylcholine and $\mathrm{Ba}^{2+}$ in normal medium (1): Tension was isometrically presented as a percent for the maximal contraction of rat uterus to $50 \mu \mathrm{M} \mathrm{ACh}$. Note a plateau-shaped type of the log dose-response curve induced by $\mathrm{Ca}^{2+}$ different from that by $\mathrm{Ba}^{2+}$, indicating that there may be a rate limiting step in the transport process of $\mathrm{Ca}^{2-}$ for exitation-contraction coupling (see text). 
responds to the minimal doses of $1 \mu \mathrm{M}$ external calcium ions for the initial response (1), the maximal response induced by $10 \mu \mathrm{M}$ ACh might require external calcium ions of $10 \mathrm{mM}$. Our result showed clearly that $10 \mathrm{mM}$ calcium ions induced only about $50 \%$ of maximal response. The influx of external calcium ions increased clearly with $\mathrm{KCl}$-depolarized medium, whereas that of barium ions decreased to about 1/1000 times. Particularly the log dose-response curve induced by calcium chloride presents a plateau around doses of $1 \mathrm{mM}$, whereas that induced by barium chloride does not.

These findings suggest that the process after influx of calcium ion through the depolarized uterine membrane may be different from that of barium ion. In the lower concentration, however, both external calcium and barium cations reach the same site of action in intracellular calcium stores.

In a dose lower than that required to produce the plateau, the contractile effects of calcium, barium and strontium ions on the depolarized uterus were compared in Fig. 2. The effective concentrations of calcium, barium and strontium chloride producing a response equivalent to $25 \%$ maximal $\mathrm{ACh}$ response were respectively $50 \mu \mathrm{M}, 760 \mu \mathrm{M}$ and $120 \mu \mathrm{M}$, Regarding contractile activity on the depolarized rat uterus, calcium ion was thus about 2.4 times more potent than strontium ion which was about 6.3 times more potent than barium ion.

Fig. 3 shows registograms in the relation between times of previously immersing uterus in Ca-free $\mathrm{KCl}$ Ringer and tensions induced by cumulative doses of calcium ion beginning with initial doses of $17, \iota \mathrm{M}$. When a recording was made after $30 \mathrm{~min}$, the relation between cumulative doses and tension was not a simple S-shape curve, rather a plateau appeared at intermediate doses. After $60 \mathrm{~min}$, however, the intermediate plateau in the cumulative log dose-response curve was slight in appearance, at the same time depression in the maximal height occurred. After $2 \mathrm{hr}$, the plateau never appeared.

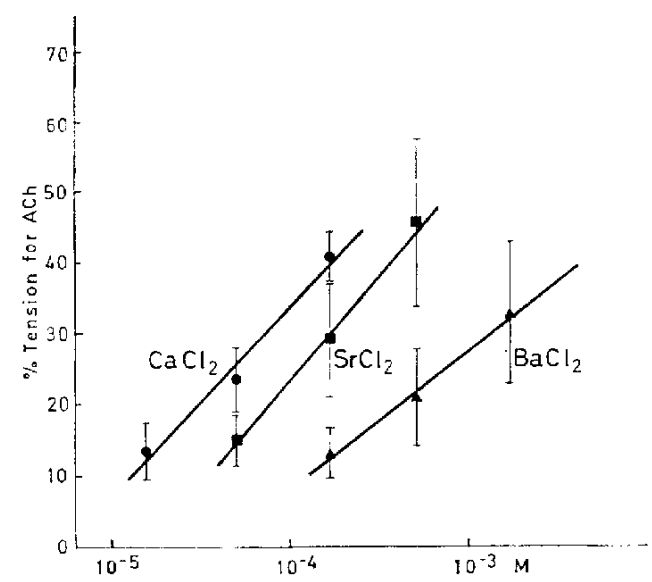

FIG, 2. The contractile effects of $\mathrm{Ca}^{2+}, \mathrm{Ba}^{2+}$ and $\mathrm{Sr}^{2+}$ on $\mathrm{KCl}$-depolarized uterus: Responses from 8 horns were averaged. Note: at the response equivalent to $25 \%$ maximal $\mathrm{ACh}, \mathrm{Ca}^{2+}$ is about 2.4 times more active than $\mathrm{Sr}^{2+}$ which is about 6.3 times more active than $\mathrm{Ba}^{2+}$. 


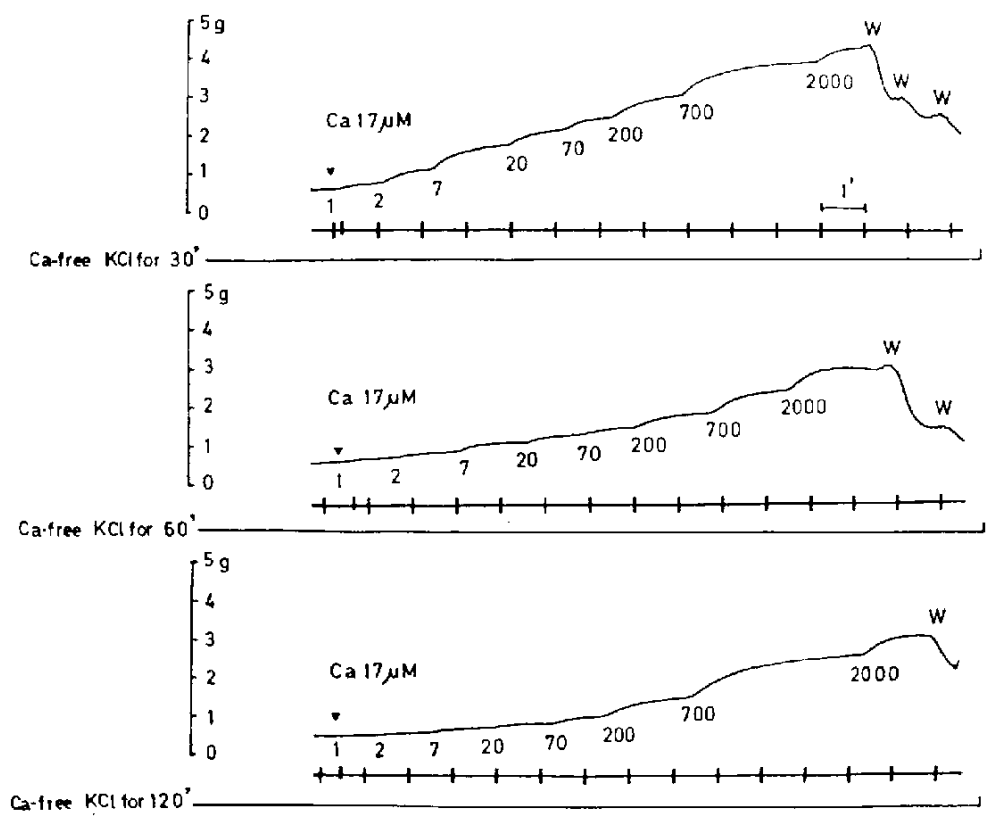

FIG. 3. Registrograms in the relation between $\mathrm{Ca}^{2+}$-induced tensions and length of time in which the uterus was immersed in Ca-frec $\mathrm{KCl}$ Ringer solution for $30 \mathrm{~min}$ (upper), for $60 \mathrm{~min}$ (middle) and for $120 \mathrm{~min}$ (under): Note the progressive decrease in tension and the diminution of the plateau in Ca-dose-response curves with immersion time. This indicates the decreasing of the effect of $\mathrm{Ca}$-induced $\mathrm{Ca}$ release, since the $\mathrm{Ca}^{2+}$ bound the internal surface of cell membrane flowed out to external phase.

Effect of magnesium ion on dose-response curve induced by calcium, barium and strontium cations: The effects of magnesium ion were studied in relation to contractile responses induced by low and high concentrations of calcium, barium and strontium cations. Typical examples are shown in Fig. 4 which illustrates some of the interactions of magnesium ion with the three cations. The interactions are complex but seem to be independent of the order of administration; the preadministration of magnesium ion producing approximately similar effects to the same dose of magnesium ion administered after contractile responses by calcium or barium ions.

Magnesium ion may antagonize or potentiate calcium ion depending on the concentration of both. Fig. 4 shows that the contractile effect of $0.2 \mathrm{mM}$ calcium ion was potentiated by $50 \mu \mathrm{M}$ magnesium ion. Magnesium ion of $20 \mathrm{mM}$ potentiated the contractile response induced by $5 \mathrm{mM}$ calcium ion. With strontium and barium ions antagonism by magnesium ion only was seen; $5 \mathrm{mM}$ magnesium ion antagonized contractile response induced by $5 \mathrm{mM}$ strontium or barium ion. A clear view of these effects was obtained by plotting log dose-response curves as shown in Fig. 5a representing data of 4 control assays (calcium alone) and 4 test assays (calcium and magnesium ion) on 4-6 horns. The curves show three phases; slight polentiation with low concentration of magnesium ion $(50 \mu \mathrm{M})$, antagonism with intermediate concentration $(0.5 \mathrm{mM})$ and antagonism followed by marked 

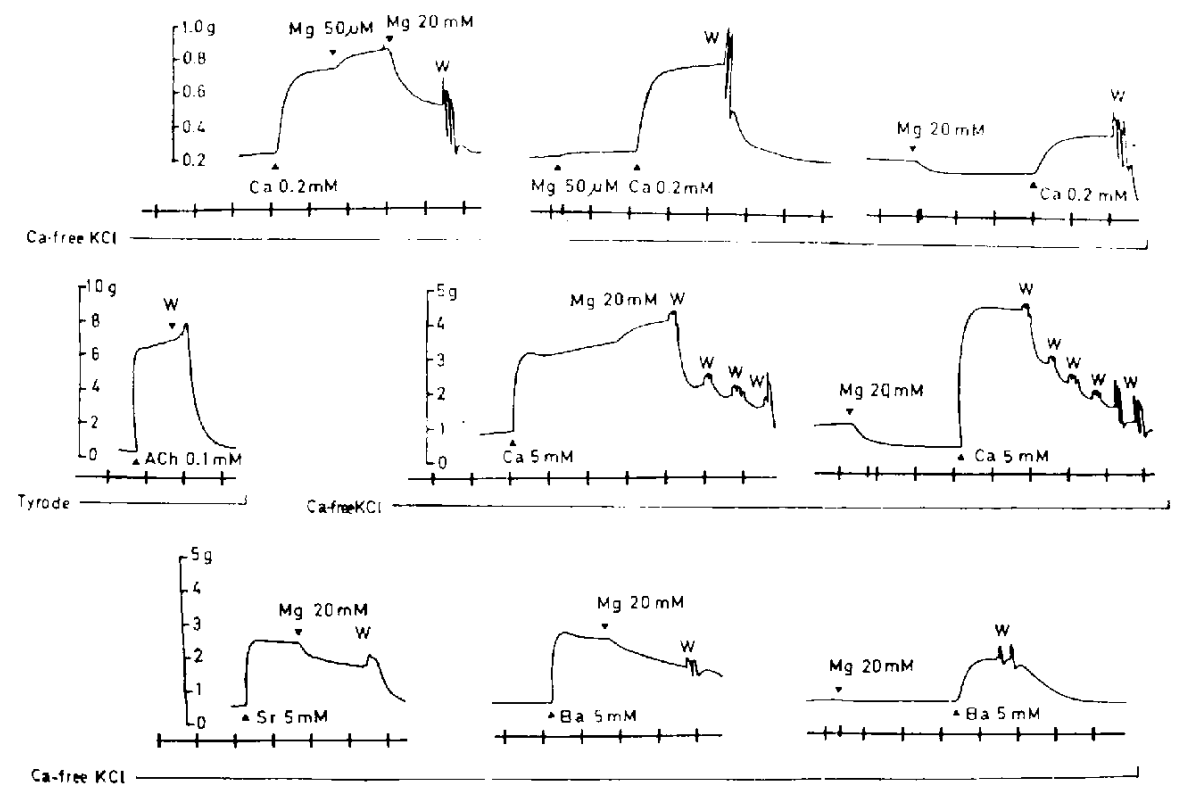

FIG. 4. Some example of records in the effects of magnesium on the $\mathrm{KCl}$-depolarized uterus contracted by $\mathrm{Ca}, \mathrm{Ba}$ and $\mathrm{Sr}$ cations: Note the same results in the effect of $\mathrm{Mg}^{2+}$ applied both before and after application of $\mathrm{Ca}^{2+}$, and the different results in the effect of $\mathrm{Mg}^{2+}$ between the contractions induced by $\mathrm{Ca}^{2+}$ and $\mathrm{Sr}^{2+}$ or $\mathrm{Ba}^{2+}$.

potentiation of maximal calcium responses by high magnesium ion $(5 \mathrm{mM})$. In contrast to those complex interactions with calcium ion, the curves of barium and strontium ions shifted only in parallel to the right in the presence of magnesium as shown in Fig. $5 \mathrm{~b}$ and $\mathrm{c}$.

The data from Fig. 5 ( $a, b$ and c) were re-plotted after the manner of Arunlakshana and Schild (4). The points lie on three nearly straight lines as shown in Fig. 6. The slopes of these regression lines are approximately 0.5 suggesting a type of competitive antagonism in which each cation of calcium, barium and strontium is antagonized by magnesium at an apparent molar ratio of $2: 1$.

Influence of manganese ion, diltiazem and dantrolene on the dose-response curve induced by external calcium ion: The modification of intermediate plateau in the log dose-response curve induced by external calcium was studied on $\mathrm{Ca}$-free $\mathrm{KCl}$ depolarized uterus under the influence of manganese ion, diltiazem and dantrolene.

Fig. 7 shows the mode of inhibitory action of three drugs on the plateau shape of log dose-response curve induced by calcium ion. The inhibitory pattern of both manganese ion and diltiazem is similar, that is strong inhibition of the intermediate plateau in log doseresponse curve induced by calcium ion and the competitive-like inhibition with a higher concentration of external calcium ion (Fig. $7 \mathrm{a}$ and b). On the other hand, dantrolene inhibited in a noncompetitive-like pattern (Fig. 7c).

The results suggest that the site of action of dantrolene may be farther from membrane 


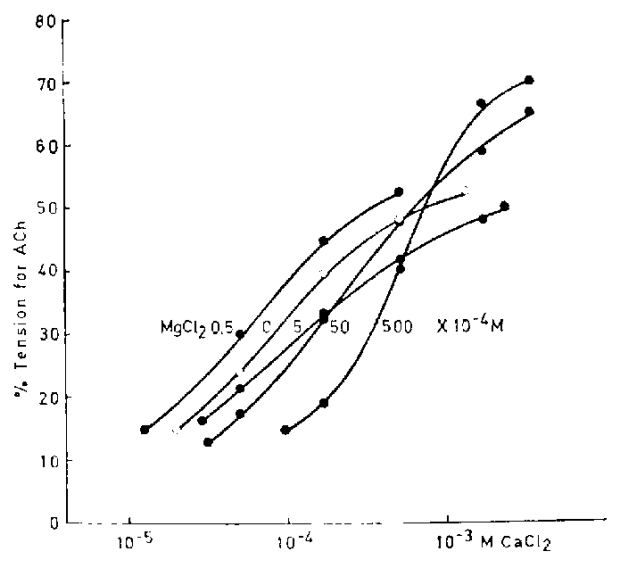

a

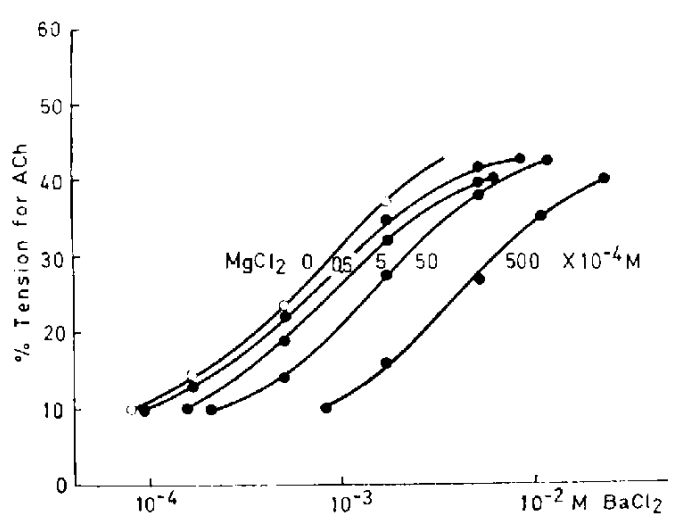

b

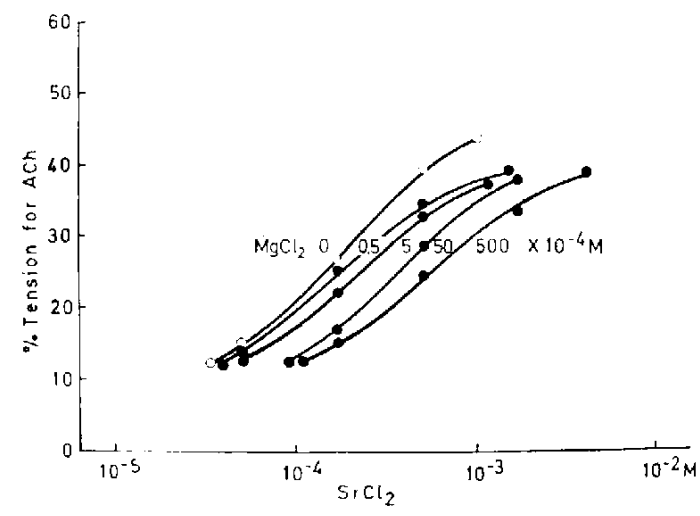

$\mathrm{c}$

FIG. 5. a) Log dose-response curves induced by $\mathrm{CaCl}_{2}$ with () or without $\mathrm{MgCl}_{2}$ $\left(0\right.$. b) Log dose-response curve induced by $\mathrm{BaCl}_{2}$, and c) Log dose-response curve induced by $\mathrm{SrCl}_{2}$ with (O) or without $\mathrm{MgCl}_{2}(\bigcirc)$.

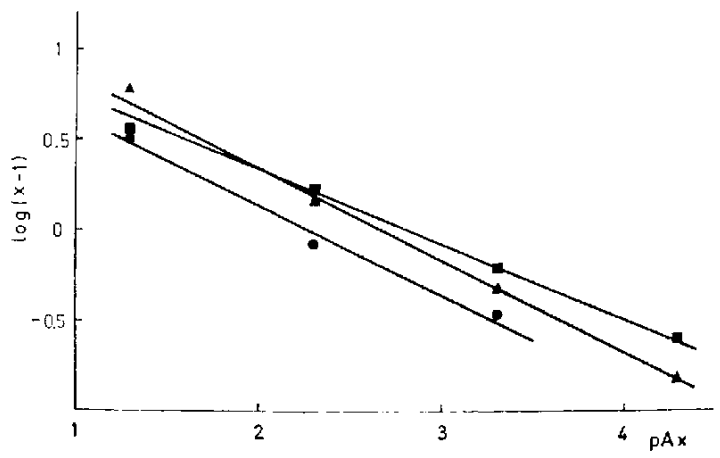

Fig. 6. Schild's plots for $\mathrm{CaCl}_{2}(\mathbf{O}), \mathrm{SrCl}_{2}(\boldsymbol{\square})$ and $\mathrm{BaCl}_{2}(\mathbf{\Delta})$ in depolarized rat uterus, using $\mathrm{MgCl}_{2}$ as an antagonist. In the case of $\mathrm{CaCl}_{2}$, dose range after the plateau was not used. Note that the slope of the Schild's plot was significantly not different from $1 / 2$ in either case of $\mathrm{CaCl}_{2}, \mathrm{SrCl}_{2}$ and $\mathrm{BaCl}_{2}$. 

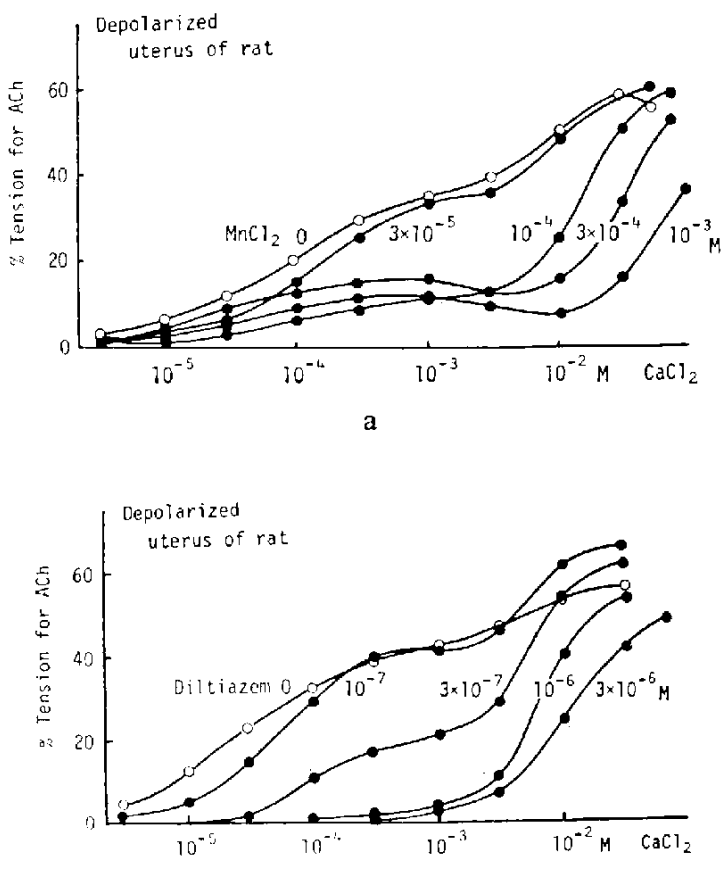

b

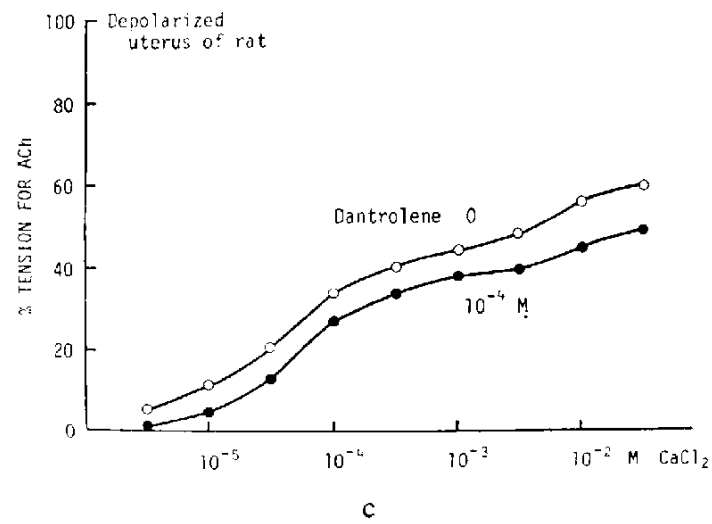

FIG. 7. Log dose-response curves induced by $\mathrm{CaCl}_{2}$ without ( $)$ and with a) $\mathrm{MnCl}_{2}$, b) diltiazem or c) dantrolene ( $)$ obtained in the $\mathrm{KCl}$-depolarized uterus. Note that both of $\mathrm{MnCl}_{2}$ of more than $10 \mu \mathrm{M}$ and diltiazem of more than $0.3 \mu \mathrm{M}$ inhibited the plateau and the maximum competitively, and that dantrolene also inhibited both of them and retained the plateau-shaped pattern.

than that of both manganese ion and diltiazem in the mechanism of excitation-contraction coupling.

Effects of EDTA and EGTA treatments on the tension of the depolarized rat uterus produced by external calcium ion: Action of external calcium ion was studied on the depolarized rat uterus treated with EDTA and EGTA. These substances are considered to 
be chemically skinned agents of skeletal and cardiac muscle fibers (5), but apparently have not yet been applied to studies on smooth muscle fibers. Fig. 8 shows the log dose-response curve induced by external calcium on the depolarized rat uterus after treatment with EDTA and EGTA solution for $30 \mathrm{~min}$ and then washed out with $\mathrm{Ca}$-free $\mathrm{KCl}$ solution for about $20 \mathrm{~min}$.

EDTA produced almost no change in the dose-response curve, whereas EGTA produced dose-dependent effects. The log dose-response curve induced by external calcium ion after treatment with EGTA changed to a simple signoid type (i.e. without the intermediate plateau) was accompanied by a potentiation of the tension, and its maximal tension induced

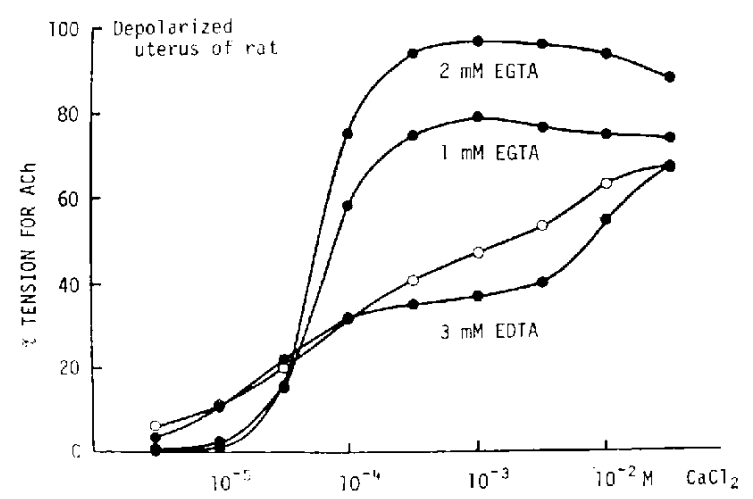

FIG. 8. Log dose-response curves induced by $\mathrm{CaCl}_{2}$ produced by treatment with $3 \mathrm{mM}$ EGTA and with 1 or $2 \mathrm{mM}$ EGTA (O) and its control (O). Note that EGTA potentiated the plateau and elicited the disappcarance of the plateau-shaped pattern.

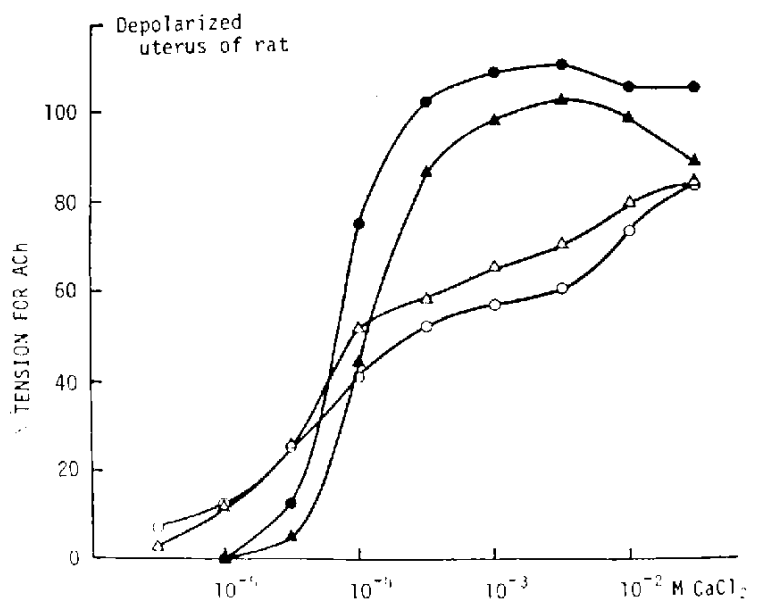

FIG. 9. Recovering of log dose-response curves induced by $\mathrm{CaCl}_{2}$ potentiated by $2 \mathrm{mM}$ EGTA one hour after washout with $\mathrm{Ca}$-free $\mathrm{NaCl}$ and $\mathrm{KCl}$ solution. Experimental design consists of two pairs, EGTA $(\bullet)$ treated after control (O), and EGTA $(\boldsymbol{\Delta})$ treated before control $(\triangle)$. Note there was no difference between the results of two pairs, irrespective of the order of treatment of EGTA and control. 
by calcium ion paralleled that induced by $\mathrm{ACh}$ on the non-depolarized muscle.

In the initial dose of cumulative log dose-response curve, on the other hand, the contractile response of EGTA treated muscle is less than that before treatment.

In the second place, the reversibility of EGTA treatment was studied. Using the uterine muscle rested for an hour after the effects of EGTA treatment, as shown in Fig. 9, the original $\log$ dose-response curve presenting the plateau was reverted to. This result suggests that EGTA with its chelating effects may deplete the calcium store where the external calcium will bind through the membrane.

\section{DISCUSSION}

The normal action of drugs on smooth muscle may be explained in terms of interaction between electrical events intimately bound up with a polarized cell membrane and mechanical events which occur independently of membrane polarization $(6,7)$. Depolarized smooth muscle preparations provide a means of studying the effects of drugs on the contractile process of smooth muscle by a mechanism which is not mediated by membrane depolarization (8). The contractile mechanism of smooth muscle has been suggested to depend on an interaction between external calcium and stores of "bond" calcium (9).

The appearance of a plateau in the log dose-response curve induced by calcium ion on $\mathrm{KCl}$ depolarized uterus can be regarded as an effect of this interaction. The evidence that the plateau is not an artifact or a misinterpretation, but a specific character of the log doseresponse curve induced by calcium ion is confirmed by the fact that change in the plateau was observed under the influence of manganese ion, diltiazem, dantrolene and EGTA. These drugs used are a kind of calcium antagonist; the mechanisms of which are the inhibition of the membrane induced by manganese (10), the interference with excitationcontraction coupling induced by diltiazem (11), and the inhibition of sarcoplasmic reticulum induced by dantrolene $(12,13)$.

In the view of the pharmacokinetical analysis for log dose-response curve, the appearance of the plateau in the log dose-response curve induced by calcium ion indicated the presence of a rate limiting step for the movement of calcium ion from external phase to a kind of calcium store. Being evidently changed by manganese ion and dittiazem which inhibit the external calcium ion entry through the membrane, the rate limiting step may relate with the cell membrane. The mechanisms may be explained by the calcium store of inner membrane. When the calcium store is depleted by the calcium chelating effect of EGTA, the external calcium may transfer to sarcoplasmic reticulum without inducing the release of calcium ion from its store. Also the observation that the intracellular calcium ions may be depleted in time course of exposition to $\mathrm{Ca}^{2+}$-free medium indicates that the extcrnal calcium ions may play an important role in compensating directly for the intracellular calcium ions. Thus the intermediate plateau in log dose-response curve induced by external calcium ion did not appear, but the maximal response increased in the same time. We concluded that the plateau of the log dose-response curve induced by calcium ion seems to be due to the rate limiting step by means of the calcium store of the inner membrane, and the curve preceded 
by the plateau is due to the high concentration of the external calcium ion bound immediately to sarcoplasmic reticulum.

According to Hurwitz (14), there is currently a consensus of view that calcium ion which can be mobilized to activate contractile apparatus in smooth muscle fibers may originatc from two different stores. One is the pool of calcium that is loosely bound to superficial sites in the muscle fiber, the other is a tightly bound pool of calcium that is sequestered in some intracellular location in the fiber. If the former corresponds to calcium stores of inner membrane, the latter may correspond to sarcoplasmic reticulum. Our observations and views are supported with this consideration.

From the views mentioned above, the mode of action of barium, strontium and manganese cations will be discussed. Effect of the contraction of $\mathrm{KCl}$-depolarized uterus was in the potency order of $\mathrm{Sr}<\mathrm{Ba}<\mathrm{Ca}$, thus being in agreement with the results reported by Bando et al. (15). In a range of low doses, the sites of action of three cations are suggested to be the same, as determined from the results shown in Fig. 6. On the other hand, the log dose-response curve induced by barium and strontium ions is different from that induced by calcium ion from the point of non-appearance of the plateau, indicating the possibility that barium and strontium ions can bind the calcium store of inner membrane (or near the membrane) to induce the release of intracellular calcium, but there is much difficult in binding to the sarcoplasmic reticulum, even at high concentrations. Barium ion on non-depolarized smooth muscle cannot enter the cellular membrane, but depolarizes it the same as ACh. The release of intracellular calcium from its store by means of the depolarization of the cell membrane is much stronger than the depletion induced by these bivalent cations on depolarized muscle.

The complex mechanisms of action of magnesium ion can be explained thus; at a low concentration, magnesium chloride induces a release of intracellular calcium ion from the calcium store of the inner membrane, whereas at the intermediate concentration, magnesium ions antagonize competitively external calcium ions. Magnesium ions may also inhibit competitively the depleting effects of the calcium store induced by barium and strontium ions. Being inhibited by the magnesium ions, however, the calcium ion in the high doses is transferred to the sarcoplasmic reticulum. Thus, the possible mechanism of action of the external calcium ion in the high concentration seems to explain adequately the appearances of the potentiation in the contracting effect of external calcium ion induced by the magnesium ion in the high doses.

It may be concluded that the site of action of the cxtcrnal calcium, barium, strontium and magnesium cations on the $\mathrm{KCl}$ depolarized uterus is the same site as in the case of the calcium store, where the antagonism and the potentiation of the external calcium ions are produced by the other bivalent cations. Also the mechanisms by which the plateau of the $\log$ dose-response curve induced by the external calcium ions produce the change, depend on the calcium store of the inner membrane.

Acknowledgements: These studies were initiated in the laboratory of Prof. Schild in University College London by one of the authors (M. K.) who was a recipient of a grant 
for study abroad from the Ministry of Education, Japan. Gratitude is due to Prof. Schild for kind hospitality and suggestions regarding this study, and to Dr. Jenkinson, for pertinent advice on part of the paper.

\section{REFERENCES}

1) SCHILD, H.O.: Calcium and the relaxant effect of isoproterenol in the depolarized rat uterus. Pharmacol. Rev. 18, 495-501 (1966)

2) SchiLd, H.O.: The action of isoprenaline in the depolarized rat uterus. Brit. J. Pharmacol. 31, 578-592 (1967)

3) Boura, A., Mongar, J.L. and SCHild, H.O.: Improved automatic apparatus for pharmacological assays on isolated preparation. Brit. J. Pharmacol. 9, 24-30 (1954)

4) Arunlakshana, O. and Schild, H.O.: Some quantitative uses of drug antagonists. Brit. J. Pharmacol. 14, 48-58 (1959)

5) Winegrad, S.: Studies of cardiac muscle with a high permiability to calcium produced by treatment with cthylenediaminctetraacetic acid. J. gen. Physiol. 58, 71-93 (1971)

6) Shibata, S. ANd Briggs, A.H.: The relationships between electrical and mechanical events in rabbit aortic strips. J. Pharmacol. exp. Ther. 153, 466-470 (1966)

7) SOMLyo, A.V. AND SOMLYO, A.P.: Electromechanical and pharmacochemical coupling in vascular smooth muscle. J. Pharmacol. exp. Ther. 159, 129-145 (1968)

8) Evans, D.H.L., Schild, H.O. and TheslefF, S.: Effects of drugs on depolarized plain muscle. J. Physiol. 143, 474-485 (1958)

9) IMAI, S. AND TAKEDA, K.: Effect of vasodilators upon the isolated tacnia coli of the guinea pig. J. Pharmacol. exp. Ther. 156, 557-564 (1967)

10) Nonomura, Y., Hotta, Y. And Ohashi, H.: Tetrodotoxine and manganese ions; Effects on electrical activity and tension in taenia coli of guinea pig. Science 152, 97-99 (1966)

11) Magaribuchi, T., Nakajima, H. and Kiyomoto, A.: Effect of diltiazem on the electrical and mechanical activities of isolated guinea pig taenia coli. Japan. J. Pharmacol. 27, 361-369 (1977)

12) YAGI, S. AND ENDO, M.: Effect of dantrolene on excitation-contraction coupling of skeletal muscle. Japan. J. Pharmacol. 26, Suppl. 164P (1976)

13) Suzuki, A. AND Hotta, K.: Effect of dantrolene sodium on skeletal, cardiac, smooth muscles and thcir sarcoplasmic reticulum. Japan. J. Pharmacol. 26, Suppl. 164P (1976)

14) Hurwitz, L.: Characterization of calcium pools utilized for contraction in smooth muscle. INSERM 50, 369-380 (1975)

15) Bando, T., Aizu, M., Sakato, M. and Yanagisawa, M.: Pharmacology of divalent ions on smooth muscle. Folia pharmacol. japon, 66, 89\$ (1970) (Abs. in English) 